\title{
VINCULACIÓN ENTRE \\ LOS ESTILOS DE APRENDIZAJE Y \\ ENSEÑANZA PARA EL LOGRO \\ DE UN APRENDIZAJE SIGNIFICATIVO
}

\author{
Ana Lizbeth Huanca Hancco
}

\begin{abstract}
RESUMEN
El presente artículo apunta a valorar algunos fundamentos teóricos y una revisión bibliográfica breve acerca de algunas conceptualizaciones de los constructos de Estilos de Enseñanza y Aprendizaje en las últimas décadas, la estrecha vinculación entre ambas variables tiene como finalidad generar una autorreflexión en los docentes acerca de sus propias prácticas pedagógicas basadas en la teoría de las diferencias individuales, para así poder garantizar una enseñanza de calidad y aprendizajes significativos. Entonces, una primera tarea del docente será identificar cuáles de los cuatro estilos de aprendizaje predomina en su grupo de clase, para adaptar su propio estilo de enseñanza de acuerdo a los estilos de aprendizaje de sus alumnos, brindándoles las herramientas y estrategias para el logro de los objetivos educativos, evitando así el fracaso escolar.
\end{abstract}

\section{PALABRAS CLAVE}

Estilos de aprendizaje, estilos de enseñanza, estrategias de aprendizaje, diferencias individuales, aprendizaje significativo.

\section{ABSTRACT}

The present article aims to evaluate some theoretical fundamentals and a brief bibliography review about some conceptualizations of the Teaching and Learning Styles constructs in the last decades, the close link among both variables has the purpose of generating a self-reflection in the teachers about their own pedagogical practices based on the theory of different individuals so as to guarantee a quality teaching and a significant learning. Furthermore, a first teacher's task will be to identify which of the four styles of learning predominates in his class group, to adapt his own style of teaching according to the students learning styles, giving them the tools and strategies for the achievement of the educational objectives, thus avoiding educational failure.

\section{KEYWORDS}

Learning styles, teaching styles, learning strategies, individual differences, significant learning.
D esde finales del siglo XX se ha observado un cambio en el antiguo paradigma ional en el que se creía que el aprendizaje era sinónimo de memorizar mecánicamente las respuestas, donde el docente era quien controlaba los contenidos que debían aprender los alumnos, es decir, basado en un sistema tradicional conductista. Por el contrario, el enfoque pedagógico actual está orientado sobre la base de las teorías constructivistas en las que el alumno es más autónomo, autorregula su propio aprendizaje y el docente se convierte más bien en un mediador del conocimiento, al que le importa no solo los contenidos sino los procesos cognitivos que promoverán en sus estudiantes a fin de lograr el éxito en diferentes ámbitos de la vida y no solo en elámbito académico.

Desde esta perspectiva, surge el interés por conocer la forma como los alumnos aprenden, cómo procesan la información, cómo resuelven problemas o actúan ante una situación de aprendizaje. En otras palabras, se habla del término "Estilos de aprendizaje", que ha sido ampliamente estudiado por diversos autores debido a su relevancia en el proceso de enseñanzaaprendizaje.

En consecuencia, para Gonzáles-Peiteado (2013) un primer paso es que el docente identifique el estilo de aprendizaje que predomina en cada alumno, para poder tomar decisiones a partir de ello, planificar las sesiones de aprendizaje, los recursos que se necesitarán, la forma de evaluación, entre otras cosas. Pero no basta con 
conocer los estilos de aprendizaje de los estudiantes, es indispensable analizarlos, replantearlos para poder reconocer las propias posibilidades y limitaciones con la finalidad de mejorar la actuación docente y ajustar su enseñanza sobre la base de las características individuales de los estudiantes.

Sin embargo, cabe también preguntarse si ¿Conocen los docentes sus propios estilos de aprendizaje y enseñanza? ¿Influyen también los estilos de enseñanza y aprendizaje de los docentes en el proceso de aprendizaje de sus estudiantes? ¿Por qué alumnos que no presentan dificultades para aprender no alcanzan los objetivos educativos?

Definitivamente cada docente posee un estilo de enseñanza propio que no siempre va en concordancia con los estilos de aprendizaje de sus alumnos, tomando en cuenta las diferencias individuales, lo cual influye rotundamente en el proceso de enseñanza-aprendizaje. En el presente artículo se trata de puntualizar la relevancia de la concordancia entre estas variables de forma cualitativa, para promover una praxis pedagógica que priorice las características particulares del educando y propiciar un proceso de enseñanza más individualizado como medio para evitar el fracaso escolar.

\section{ANÁLISIS}

\section{Estilos de Aprendizaje}

Para hablar sobre los Estilos de Aprendizaje se realizará una aproximación conceptual, debido a las diversas teorías y enfoques del cual es objeto de estudio, los que contribuyen a entender los comportamientos que se observan en el aula, cómo se relacionan estos con la forma como aprenden los estudiantes y cuáles son las alternativas de actuación docente más eficaces en una situación o circunstancia dada (Ventura 2011).

Se podría decir que los primeros estudios acerca de estilos de aprendizaje surgieron desde la década de los setenta, sin embargo, es a finales del siglo XXI en que se puso mayor énfasis en las teorías constructivistas que iban de la mano con las diferencias individuales que influían en el proceso de enseñanza-aprendizaje. En 1981, Schuel definió a los Estilos de Aprendizaje como aquellas formas preferidas por la que los estudiantes procesan y organizan la información, en respuesta a los estilos del ambiente (Quiñones 2004).

Asimismo, Alonso, Gallego y Honey (1994) explican que los Estilos de Aprendizaje son "los rasgos cognitivos, afectivos y fisiológicos que sirven como indicadores relativamente estables, de cómo los discentes perciben, interrelacionan y responden a sus ambientes de aprendizaje".

Es importante considerar los rasgos cognoscitivos del alumno, ya que permite al docente conocer algunos aspectos esenciales de la inteligencia como aquellas estrategias de procesamiento de información, habilidades y aptitudes de sus alumnos; todas ellas variables complejas que explican las diferencias individuales entre el alumnado. La dimensión afectiva, del mismo modo, incide notablemente en la configuración de los estilos aprendizaje, en el sentido en que los aprendizajes están fuertemente relacionados a las motivaciones y afectos, los cuales facilitaran conducir a alcanzar los objetivos educativos. Por tanto, si el alumno no está suficientemente motivado su aprendizaje será deficiente, pudiendo derivar en fracaso (González 2005). Igualmente, es preciso tener en cuenta los intereses y las expectativas.

Por otro lado, los aspectos de orden fisiológico, que tienen una base física e igualmente, reflejan las diferencias en el modo en que un individuo responde ante las situaciones según el género, la edad y el contexto de aprendizaje. Atendiendo a este último rasgo, existen personas que estudian mejor por la mañana, por la tarde o por la noche. Otros factores como los elementos contextuales, entre los que destacamos, tipo de organización, iluminación, ruido y espacio, también tienen incidencia en el modo en que un sujeto va a construir su proceso educativo.

Al hablar de enfoques se puede observar en la literatura una gran diversidad de autores que plantean teorías sobre los Estilos de Aprendizaje. Ventura (2011), realizó una recopilación de los principales enfoques teóricos hasta la actualidad: 
Tabla 1

Enfoques teóricos principales de los estilos de aprendizaje

\begin{tabular}{|c|c|c|}
\hline Máximos representantes & Categorías de análisis & Instrumentos \\
\hline $\begin{array}{l}\text { Schmeck,, Ribich } \\
\text { y Ramanaiah } \\
\text { (1977) }\end{array}$ & $\begin{array}{l}\text { Procesamiento profundo } \\
\text { Procesamiento elaborativo } \\
\text { Memoriade hechos } \\
\text { Estudio metódico }\end{array}$ & $\begin{array}{l}\text { Inventory of learning Processes } \\
\text { (ILP) }\end{array}$ \\
\hline Briggs-Myers (1978) & $\begin{array}{l}\text { Extroversión-introversión } \\
\text { Sensación-intuición } \\
\text { Pensamiento-sentimiento } \\
\text { Juicio-percepción }\end{array}$ & $\begin{array}{l}\text { Indicador de Tipos Psicológicos } \\
\text { Briggs-Myers (MBTI) }\end{array}$ \\
\hline Dunn, Dunn y Price (1983) & $\begin{array}{l}\text { Diseñódel ambiente } \\
\text { Emocionalidad personal } \\
\text { Necesidades sociales } \\
\text { Necesidades fisiológicas }\end{array}$ & $\begin{array}{l}\text { Learning Styles Inventory } \\
\text { (LSI) }\end{array}$ \\
\hline Kolb (1984) & $\begin{array}{l}\text { Divergentes } \\
\text { Asimiladores } \\
\text { Acomodadores } \\
\text { Convergentes }\end{array}$ & $\begin{array}{l}\text { Inventario de Estilos de Aprendizaje } \\
\text { (IEA) }\end{array}$ \\
\hline Feldery Silverman (1988) & $\begin{array}{l}\text { Activo-reflexivo } \\
\text { Sensorial - intuitivo } \\
\text { Visual-verbal } \\
\text { Secuencial-global }\end{array}$ & $\begin{array}{l}\text { Index of learning Styles } \\
\text { (ISL) }\end{array}$ \\
\hline Honeyy Alonso & $\begin{array}{l}\text { Activo } \\
\text { Reflexivo } \\
\text { Teórico } \\
\text { Pragmático }\end{array}$ & $\begin{array}{l}\text { Cuestionario de Honey y Alonso de } \\
\text { Estilos de Aprendizaje (CHAEA) }\end{array}$ \\
\hline
\end{tabular}

Fuente: Tomado de Ventura (2011)

De todos estos enfoques, se tomará como referencia para el presente estudio teórico, el último, debido a que es la perspectiva con mayor auge en la actualidad dentro del contexto latinoamericano, especialmente en Chile, Venezuela, Colombia y Perú (Bolívar y Rojas 2008).

Honey y Alonso se basaron en los aportes realizados por Honey y Mumford (1986), quienes en un primer momento adoptan algunas ideas de Kolb (1984); sin embargo, éstos replantean los estilos de aprendizaje y crean un instrumento propio (CHAEA), el cual define cuatro estilos de aprendizaje: activo, reflexivo, teórico y pragmático (Ventura 20ll).

Tabla 2

Estilos de aprendizaje

\begin{tabular}{|cl|}
\hline Estilo & \multicolumn{1}{c|}{ Característica } \\
\hline Activos & $\begin{array}{l}\text { Estas personas se implican en los asuntos de los demás y centran a su } \\
\text { alrededor todas las actividades que emprenden con entusiasmo. Son de } \\
\text { mente abierta, nada escépticos. Sus dias están llenos de actividad. }\end{array}$ \\
& $\begin{array}{l}\text { Piensan que por lo menos una vez hay que intentarlo todo. Espontáneos, } \\
\text { creativos, innovadores, deseosos de aprender y resolver problemas. }\end{array}$ \\
\hline Reflexivos & $\begin{array}{l}\text { Gustan considerar las experiencias y observarlas desde diferentes } \\
\text { perspectivas, reúnen datos analizándolos con detenimiento antes de llegar }\end{array}$ \\
& a alguna conclusión. Son prudentes, analizan bien y consideran todas las \\
alternativas posibles antes de realizar un movimiento. Escuchan a los \\
demás y no actúan hasta apropiarse de la situación, son ponderados, \\
pacientes, inquisidores, lentos y detallistas.
\end{tabular}
Tomado de Alonso, Gallegos y Honey (1994).

\section{Estilos de enseñanza}

El análisis de las prácticas docentes conlleva a la búsqueda de estilos eficaces para identificar aquellos que son más significativos. Por lo que ha surgido otra extensión del concepto de estilos pero dentro del área de enseñanza, dicho concepto ha sido adoptado para referirse a las diferencias en la forma de enseñar de los docentes (Rendón 2013).

Con relación a la delimitación conceptual del término estilos de enseñanza, no se halla una sola propuesta integradora, ya que existen diversos factores y variables influyentes que consideran distintos autores al momento de definirlo.

Cabe resaltar que existen diversas acepciones similares a Estilos de Enseñanza que son tomadas por sinónimos de la variable, de acuerdo a la conceptualización de algunos autores; por ejemplo, método o forma de enseñanza, modelo didáctico o docente, práctica docente, enfoque, quehacer docente, entre otras muchas denominaciones (Rendón 2013).

Rendón también realiza una compilación de una gran cantidad de conceptualizaciones realizadas por diversos estudiosos, entre las cuales se mencionará las más representativas, como la de Bennet (1979) quien afirmaba que los estilos de enseñanza era "la forma peculiar que tiene cada profesor de elaborar el programa, aplicar el método, organizar la clase y relacionarse con los alumnos, es decir, el modo de llevar la clase".

Por otro lado, Guerrero (1988) lo define como "el conjunto de actitudes y acciones sustentadas y manifestadas por quien ejerce la docencia, expresadas en un ambiente educativo definido y relativas a aspectos tales como relación docente alumno, planificación, conducción y control del proceso de enseñanza aprendizaje".

Asimismo, Grasha (1996) conceptualiza a los estilos de enseñanza como aquel conjunto de necesidades, creencias y comportamientos que los profesores preuniversitarios expresan en el aula de clase. De acuerdo a su multidimensionalidad, los estilos del docente en particular afectan la forma como interactúan con los estudiantes, dirigen las tareas en el aula, supervisan asignaciones, 
socializan con los estudiantes y los orientan.

Una definición más reciente, es la de Gonzáles (2010) que lo refiere como el modo particular de enseñar, la tendencia docente de adoptar un determinado modo de interactuar con el alumno en función de las demandas específicas de la tarea y las necesidades percibidas del educando.

Como líneas arriba se describe, no solo existe una diversidad de acepciones para el constructo de estilos de enseñanza sino que también se recoge una variedad de componentes que forman parte de la variable según cada autor; por ejemplo, en el caso de Bennet (1979) que considera que son cuatro los componentes: Método, Modo de llevar la clase, Organización de la clase, Relación con los alumnos. De otro lado, Guerrero (1988) halla además otros componentes más como: Ambiente educativo, Concepción docente de la educación, Conducción, Control del proceso de enseñanzaaprendizaje, Planificación, Relación docentealumno y Tipo de acción ejecutada para lograr el fin. Y de la misma forma cada autor ha planteado distintos componentes que influyen en la práctica docente.

Sin embargo, tomando en cuenta que los estilos de los docentes tienen un carácter individual y singular, que además se articulan a factores de tipo social y cultural, se complejiza poder establecer un solo modelo o estilo de enseñanza pedagógica que vaya acorde a la individualidad de cada estudiante.

Valdivia (2002) y Freitas (2012) señalan que sí es importante que los docentes adopten su etilo de enseñanza de acuerdo a la forma de aprender del alumnado, es decir, previo a ello debe tener un conocimiento de los estilos de aprendizaje de sus estudiantes.

En concordancia con esta idea, Kolb (1976) desarrolla un modelo de enseñanza compatible con cada estilo de aprendizaje, desde este enfoque, se recomienda que los profesores desarrollen diversos estilos de enseñanza para poder aplicar distintas estrategias para conectar con todos sus estudiantes sea cual fueren sus estilos de aprendizaje (Gonzáles-Peiteado 2013).

En contraparte existen algunos autores que no están de acuerdo con esta idea, sino que por el contrario afirman que el docente debe ayudar a sus estudiantes a desarrollar los otros estilos de aprendizaje menos predominantes brindándoles las técnicas y estrategias necesarias.

\section{CONCLUSIONES}

La presente revisión documental acerca de las variables Estilos de aprendizaje y Estilos de enseñanza, nos permitió revisar en principio algunos conceptos para poder establecer un vínculo entre ambos constructos, encontrándose aún ciertas ambigüedades en las conceptualizaciones, sin embargo varias investigaciones han demostrado que el proceso de aprendizaje se facilita cuando el docente enseña de acuerdo al estilo preferente del estudiante, hallándose entonces una relación significativa entre estas variables, lo que contribuye en lograr un aprendizaje significativo en los estudiantes.

Partiendo de lo anterior, es imprescindible que todos los maestros conozcan los estilos de aprendizaje que predominan en sus estudiantes, de tal forma que puedan adaptar las estrategias y formas de enseñanza de acuerdo a ellas, favoreciendo el aprendizaje centrado en el alumno yel logro de los objetivos educativos.

En el contexto latinoamericano suele predominar el estilo expositivo oral, es decir, lo auditivo está presente en un 90\% de las clases. Pese a que ya hemos entrado en un cambio de paradigma educacional, aún persisten algunos rezagos de la enseñanza tradicional, esto se da muchas veces porque aun habiendo identificado los estilos de aprendizaje, los docentes en ejercicio no se encuentran lo suficientemente capacitados para utilizar diversos recursos y estrategias de llegada a sus alumnos acorde a los estilos de aprendizaje que presentan.

Es menester entonces, que primero el docente identifique cuál es su propio estilo de aprendizaje y enseñanza, y en base a su propia meta-análisis destaque la reflexión para conseguir un cambio en su práctica docente y un proceso de enseñanza más individualizado con el fin de evitar el fracaso escolar y promover aprendizajes que sean útiles no solo para el contexto académico sino para la vida en general. 


\section{REFERENCIAS}

Alonso, C.; Gallego, D; Honey, P. (1994). Los Estilos de Aprendizaje: Procedimientos de diagnóstico y mejora. Bilbao: Ediciones Mensajero.

Bolívar, J. y F. Rojas (2008). Los estilos de aprendizaje y el locus de control en estudiantes que inician estudios superiores y su vinculación con el rendimiento académico. Investigación y Postgrado, 23 (3), pp. 199-215.

Castro, S. y Guzman, B. (2005). Los estilos de aprendizaje en la enseñanza y aprendizaje: Una propuesta para su implementación. RevistadeInvestigación, 58 (1), pp. 83-102.

Gallego, J. (2004). Enseñar a pensar en la escuela: El aprendizaje. Madrid: Ediciones Pirámide.

Gonzáles-Peiteado, M. (2013). Los estilos de aprendizaje y enseñanza como soporte de la actividad docente. Revista de Estilos de Aprendizaje, 11 (11), pp. 17-28.

Lozano, A. (2005). Estilos de aprendizaje y enseñanza. Mad. Sevilla.

Rendón, M. (2013). Hacia una conceptualización de los estilos de enseñanza. Revista ColombianadeEducación, 64 (1), pp.175-195.

Quiñones, C. (2004). Metodología de enseñanzaaprendizaje y estilos de aprendizaje. Umbral-Revista de Educación, Cultura y Sociedad, 4(6), pp. 48-6l.

Valdivia, F. (2002). Estilos de aprendizaje en la EducaciónPrimaria. Málaga: Dykinson.

Ventura, A. (2011). Estilos de aprendizaje y prácticas de enseñanza en la universidad. Un binomio que sustenta la calidad educativa. Perfiles Educativos, 33 (1). 